\title{
Development of Real-time Measurement of effective Dose for High Dose Rate Neutron Fields
}

\author{
Final report \\ FG07-01ID14103
}

\author{
L. A. Braby, W. D. Reece and W. H. Hsu \\ Texas Engineering Experiment Station \\ College Station Texas
}

This material is based upon work supported by the U S Department of Energy under Award No. FG07-01ID14103

Any opinions, findings, and conclusions or recommendations expressed in this material are those of the authors and do not necessarily reflect the views of the Department of Energy. 
Executive summary: Studies of the biological effects of neutrons, and other applications of neutron irradiation facilities, require both a source of neutrons and a way of characterizing the radiation exposure. In the case of neutrons and other high LET radiations, the dose (energy deposited per unit mass) is not sufficient to describe the exposure, since most effects of this radiation depend on the "quality" of the radiation as well as the energy deposited. No ideal way of specifying radiation quality has yet been found, but neutron energy, the linear energy transfer of the secondary radiation, and the lineal energy have been used in formulating different definitions of quality. Depending on the source of the radiation it may be relatively easy to calculate the neutron energy spectrum, or it may be very difficult. It is almost always difficult to measure the neutron spectrum. If the Neutron spectrum can be evaluated, then the LET distribution can be calculated, but it can not be measured directly. The easiest of the three quantities to measure is the lineal energy. At low dose rates, where individual energy deposition events (passage of a secondary particle through a small volume) can be measured, the probability density of lineal energy can be measured directly and average values such as the dose mean lineal energy can be calculated. However, most experiments require dose rates that are two high to measure as individual energy deposition events in detectors of reasonable size.

The variance method provides an alternative way of measuring the dose mean lineal energy, without having to measure individual events. This method is based of measuring the total dose deposited in a specified time interval. This dose is the sum of the energy deposited by each interaction occurring during that time, and because the number of events is relatively small, the energy measured in identical time intervals will vary. The variance of the dose will depend on the size and number of individual events producing the dose, the larger the individual events, the greater the variance. It can be shown that the dose mean lineal energy can be calculated from the measured variance of dose in identical samples if the source dose rate is constant.

The electronics and detectors necessary to measure the dose and dose mean lineal energy in mixed neutron and gamma ray radiation fields with dose rates in excess of 1 $\mathrm{Gy} /$ minute have been developed. This is the dose rate that would typically be used for radiation biology experiments using filtered radiation from a research reactor. To achieve this capability, a specialized current integrator which produces output pulses proportional to the dose received in a specified interval (typically $100 \mathrm{~ms}$ ) and small spherical ion chambers, $4.3 \mathrm{~mm}$ in diameter, were developed. The output of the current integrator was processed by a multichannel analyzer and software to calculate the mean and variance of the charge. The ion chamber is operated at or below atmospheric pressure to simulate tissue volumes ranging from a few nanometers to a few micrometers in diameter.

This system has been used to measure dose and dose mean lineal energy for radioactive sources and for reactor neutron fields at high dose rate. The results are consistent with calculated from single event data measured at much lower dose rates in radiation fields which approximate the spectra of the high dose rate fields needed for biology experiments. 
Introduction: The reactor irradiation cell at the Nuclear Science Center, Texas A\&M University can provide a mixed neutron-gamma radiation field of high dose rate for a variety of biological experiments such as animal and cell tissue irradiations. A specific neutron fluence distribution and dose rate needed can be achieved by filtration and scattering through combinations of different filters, which will change the radiation quality at the irradiation target. Radiation quality is used to characterize differences in the effectiveness of various radiations when the dose and dose rate are kept constant. Determining radiation quality at the target point such as rat's lung is therefore important for comparing experiment results. For a constant radiation source, the dose variance in a small volume during a fixed period of time is proportional to the dose-mean linear energy $(\mathrm{keV} / \mu \mathrm{m})$, a microdosimetric measure of radiation quality. [1] A detection system for this project was developed to measure the dose mean lineal energy for various simulated tissue volumes (tens of $\mathrm{nm} \sim \mathrm{a}$ few $\mu \mathrm{m}$ ) at high dose rate in real-time using the variance method.

Materials and Method: The system includes three major units: a microdosimetric detector (operated in the current mode) which can simulate a small volume of tissue, an electronic unit for collecting charge for a fixed period of time from the microdosimetric detector, and a multi-channel analyzer (MCA) coupled to a computer for variance analysis and data processing.

The microdosimetric detector consists of a spherical tissue-equivalent ion chamber in the diameter of $0.43 \mathrm{~cm}$ ( $0.17 \mathrm{in})$, and a thermal couple for monitoring the detector temperature, mounted inside an aluminum vacuum container as shown in Fig. 1. The ion chamber was built by assembling two identical A-150 tissue-equivalent plastics cylinders with hemispherical depressions. The plastic cylinders provide a minimum wall thickness of $2 \mathrm{~mm}$ for the detector cavity. The center conductor of a coaxial cable forms the anode. The container was designed so that the detector can be placed in a phantom rat's body and operated with gas pressure of a fraction of one atmosphere. The temperature reading from the thermal couple and the gas pressure in the container will determine the gas density in the ion chamber and, therefore, the simulated tissue volume. [1]

The electronic unit, based on a commercial field effect transistor and integrated circuits (ICs) [2]. With the voltage applied to the transistor gate holding it in the off condition, the charge from the ion chamber is collected on the source/drain capacitance of the FET. A pulse from an integrated circuit timer causes the transistor to temporarily conduct, forming a pulse which is proportional to the charge that had been collected on the transistor. The pulse is amplified by a second integrated circuit, and the resulting pulse is applied to the multi channel analyzer, MCA. By setting up the auto-run function in the MCA, identical measurements can be repeated and the data stored in the computer. FORTRAN programs were written to calculate dose, variance, and dose mean specific energy from the MCA data 


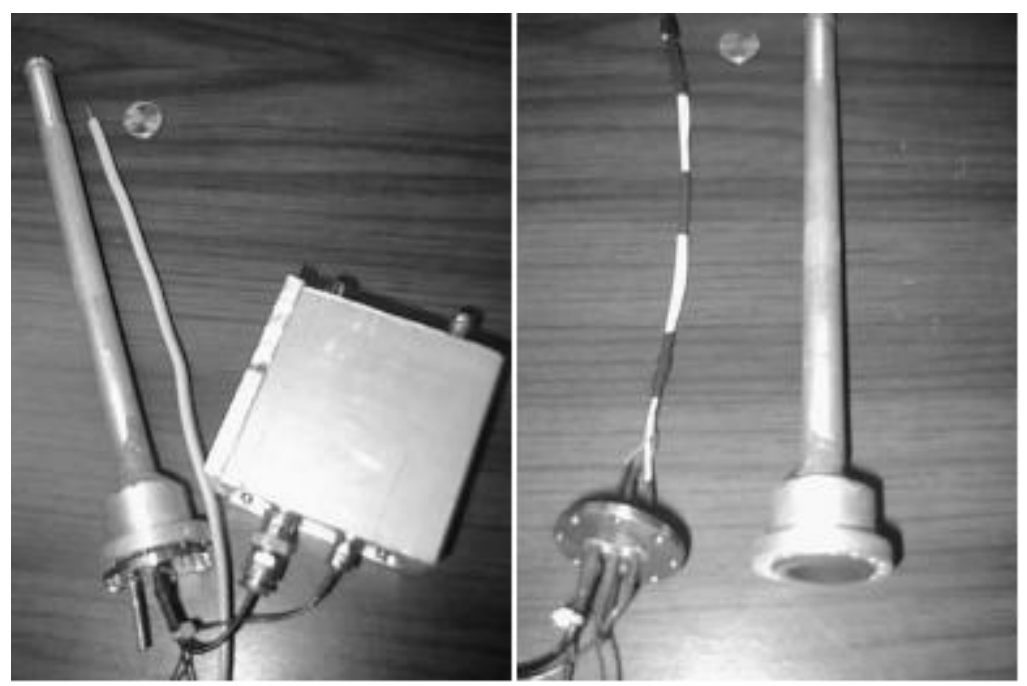

Fig. 1. The microdosimetric detector consists of a mini spherical tissue-equivalent ion chamber, an Aluminum container and a thermal couple.

Charge integrator: For the dose rates likely to be encountered in radiation biology exposure facilities, and for the detector sizes appropriate for use in most exposure facilities, variance measurements can best be made with a charge integration time on the order of 0.1 seconds. Several charge integrating circuits, suitable for integration times in this range, were evaluated. The result was that the most satisfactory was also very simple and inexpensive. It is based on integrating the charge on the capacitance of a FET, and type NTE326 was found to work well for the purpose. An integrated circuit timer, SPG8651A, was used to generate gate pulses at $100 \mathrm{~ms}$ intervals, and an operational amplifier, LT1793, is used to amplify the voltage pulse that is produced across a $1 \times 10^{6}$ ohm resister from the FET source to ground. This simple system proved to be useful for integration times ranging from 10 to $500 \mathrm{~ms}$, with very good linearity and leakage current noise of $2.3 \%$ at $1.28 \mathrm{x} \mathrm{ao}^{-13}$ amps. Because of the digital nature of the reading process, the average leakage current is easily subtracted from the result.

Test chamber: For initial tests of the charge integrator and variance method, an existing small ion chamber was used. This chamber was a $6 \mathrm{~mm}$ long, $3 \mathrm{~mm}$ diameter cylinder with hemispherical end and $0.9 \mathrm{~mm}$ diameter anode. Although the complex path length distribution in this volume, and the lack of provisions for operation below atmospheric pressure limited the use of this detector, it provided the opportunity to test the rest of the system while the spherical, low pressure detector described above was being assembled.

Results: Using the cylindrical test chamber, which simulated an annular site with a mean chord length of 3 micrometers, variance measurements were made in a ${ }^{137} \mathrm{Cs}$ irradiator, in X-ray fields at $100,140,200$, and $240 \mathrm{kV}$, and in mixed neutron and gamma fields with and without an added $4 \mathrm{~cm}$ water moderator. In these different fields, the measured dose rates varied from 0.08 to $0.9 \mathrm{~Gy} /$ minute, and were consistent with other measurements of the dose rate at the same locations. Measured values of the dose mean 
lineal energy ranged from $1.10 \mathrm{keV} / \mu \mathrm{m}$ to $5.129 \mathrm{keV} / \mu \mathrm{m}$. Because the chord length distribution of this detector has not been used for any previous measurements, these values can not be compared with previous data. However, these results are consistent with values expected for these radiations, and decrease with increasing photon energy, as expected.

Using the $4.3 \mathrm{~mm}$ diameter detector, the dose rate and radiation quality of the Cs-137 calibration source and the corresponding parameters, measured at different times, are listed in Table 1 . The air density was calculated from the temperature, which determined the simulated tissue volume. There was $1 \%$ difference of simulated tissue volume due to the temperature difference. The thermal couple will be very useful while conducting the reactor irradiation cell measurements because the temperature depends on the reactor power. The 5\% difference of dose rate was due to the different detector position in the Cs-137 calibration chamber. The measured dose mean linear energies, theoretically independent of the dose rate, are close to the value listed in the literature. [1] The detection system, inexpensive and portable, proved to be a reliable tool to measure the radiation quality of an irradiation field. The radiation qualities in the phantom rats lung are to be measured in the reactor irradiation cell with various filtration and scattering setups.

Table 1. Dose rate and radiation quality of the Cs-137 Calibration source and the corresponding parameters measured at different times.

$\begin{array}{lll}\text { Dose rate }(\mathrm{Gy} / \mathrm{s}) & \begin{array}{l}\text { Day } 1 \\ 1.74 \times 10^{-3}\end{array} & \begin{array}{l}\text { Day } 5 \\ 1.82 \times 10^{-3} \\ \pm 1.01 \times 10^{-5}\end{array} \\ \begin{array}{l}\text { Simulated tissue volume } \\ \text { diameter }(\mu \mathrm{m})\end{array} & 3.50 & 3.54 \\ \begin{array}{l}\text { Dose mean linear energy } \\ (\mathrm{keV} / \mu \mathrm{m})\end{array} & 1.25 \pm 0.55 & 1.20 \pm 0.53 \\ \begin{array}{l}\text { Temperature }\left({ }^{\circ} \mathrm{C}\right) \\ \text { Air density }\left(\mathrm{g} / \mathrm{cm}^{3}\right)\end{array} & 20.3 & 16.2 \\ & 1.22 \times 10^{-3} & 1.23 \times 10^{-3}\end{array}$




\section{References}

1. International Commission on Radiation Units and Measurements. (ICRU) Microdosimetry. International Commission on Radiation Units and Measurements. ICRU Report 36; 7910 Woodmont Ave., Bethesda, MD 20814; 1983.

2. W. Hsu, L.A. Braby, W.D. Reece, Electronics For Measuring Radiation Quality at High Dose Rate Using The Variance Method, American Nuclear Society 2002 Annual Meeting, Hollywood, Florida, USA.

\section{Publications}

Hsu, W. H., Detectors and Electronics for Real Time Measurement of Radiation Dose and Quality Using the Variance Method, Dissertation submitted to Texas A\&M University 2002

W. Hsu, L.A. Braby, W.D. Reece, Electronics For Measuring Radiation Quality at High Dose Rate Using The Variance Method, Transactions of the American Nuclear Society $86,196,2002>$

Hsu, W.H., L. A. Braby, and W. D. Reece, Detection system for Measuring Radiation Quality in the Phantom Rats Lung Using the Variance Method, Transactions of the American Nuclear Society 88, 350-351, 2003> 